\title{
Código de Procedimientos de Evaluación y Tratamiento de Terapia Ocupacional: Una Propuesta
}

Alicia Trujillo Rojas, M.A., OTR Profesora Asociada y Emérita Universidad Nacional de Colombia

El propósito de este artículo es presentar un código de procedimientos para terapia ocupacional, que sea universal, es decir aplicable a todas las áreas de ejercicio y que pueda ser empleado para rendir cuentas sobre los servicios ante diversos interlocutores. La intención de la autora es que el texto sea analizado y puesto a prueba por parte de los miembros de la comunidad profesional, para alcanzar una fase posterior de consenso, basado en los ajustes a que de lugar.

Disponer de una herramienta como la que aquí se presenta, para referirse a los programas de atención, es una necesidad sentida en el ejercicio de la terapia ocupacional en Colombia, por cuatro razones. En primer lugar, permite explicar acerca de los programas a los usuarios de los servicios; a otros colegas y estudiantes; a diversos profesionales; a personas encargadas de la gestión administrativa y financiera; y a instancias responsables de la legislación y la generación de política local, regional y nacional. En segundo lugar, contribuye al consenso en terminología por parte de la comunidad profesional. En tercer lugar, es instrumental para la gerencia de servicios. $Y$ en cuarto lugar, es útil para el desarrollo de proyectos investigativos.

Tener sistemas que identifiquen el hacer de una profesión y contar con lenguaje uniforme son algunos de los criterios que en sociología ocupacional

\footnotetext{
* Trujillo R.A. (1998) Código de procedimientos de evaluación-tratamiento de terapia ocupacional.
} 
se emplean para estimar el grado de profesionalización de un grupo ocupacional. En el contexto internacional se conocen dos aportes que aunque no precisan los procedimientos de terapia ocupacional, sí son valiosos para su diseño. Un aporte es el sistema de terminología uniforme producido en los Estados Unidos, que captura la esencia de la terapia ocupacional y permite la documentación de los servicios (American Occupational Therapy Association, 1994). Otra contribución es un texto canadiense que ofrece directrices para el ejercicio profesional, incluyendo la clasificación y definición de los conceptos esenciales para el ejercicio (Canadian Association of Occupational Therapists, 1991).

En Colombia no se han diseñado aún instrumentos similares a los producidos en norteamérica. Este vacío es una amenaza para la comprensión social y posicionamiento de la terapia ocupacional porque no permite describir los servicios de manera clara, ágil y completa, cuando diversas fuentes requieren esa identificación. Por ejemplo, la Resolución \#5261 de 1994 del Ministerio de Salud, por la cual se establece el Manual de Actividades, Intervenciones y Procedimientos del Plan Obligatorio de Salud sólo identifica de manera ambigua, incompleta e imprecisa los procedimientos de terapia ocupacional (Artículos \#84 y \# 109). O el instrumento de la Superintendencia Nacional de Salud (1997) para verificar los requisitos esenciales de los servicios de salud, en lo que hace a terapia ocupacional, no denota poseer información adecuada ni suficiente sobre las exigencias y procedimientos de este quehacer profesional.

El método empleado para la elaboración de la propuesta que aquí se presenta se basa en una reflexión sistemática, fundamentada en: (a) el postulado que formula la autora sobre su propio carácter de experta en el ejercicio de la terapia ocupacional; (b) la revisión de la literatura internacional; y (c) la consulta a historias de programas de terapia ocupacional en diversos campos de actuación. 
El código de procedimientos que aquí se comparte con la comunidad profesional, se encuentra en la primera fase de construcción. Hacia adelante será necesario proceder a la validación del contenido, a través de la consulta a expertos y de la prueba empírica en estudios de campo. Adicionalmente, sería recomendable que los lectores de este artículo puedan hacer llegar sus comentarios a la Revista Ocupación Humana, en la Sección "Cartas al Editor» o a la dirección de la autora: Facultad de Medicina, oficina 523, Universidad Nacional de Colombia, Santa Fe de Bogotá.

\section{PROCEDIMIENTOS DE EVALUACIÓNYTRATAMIENTO}

Procedimientos son los patrones de práctica que emplean los representantes de una profesión y a través de los cuales les reconoce la sociedad. En terapia ocupacional, procedimientos son todas aquellas estrategias, métodos y medios que distinguen el ejercicio profesional y que son instrumentales para llevar a cabo un programa de atención a usuarios. Los procedimientos que aquí se presentan aparecen bajo un sistema de codificación numérica, de manera que esta nomenclatura pueda ser empleada con fines de tabulación y cruces analíticos de los datos. Los procedimientos se agrupan en: (a) procedimientos de evaluación y (b) procedimientos de tratamiento/intervención.

\section{PROCEDIMIENTOS DE EVALUACIÓN}

Los procedimientos de evaluación hacen referencia a las estrategias y métodos que permiten: (a) determinar el estado pasado, presente y futuro del desempeño ocupacional de una persona, en lo relacionado con capacidades y discapacidades y con restricciones del medio ambiente fisico y social; (b) definir la elegibilidad del usuario para un programa de tratamiento; y (c) determinar expectativas de cambios asociados a la aplicación del tratamiento/ intervención.

Los procedimientos de evaluación se clasifican de acuerdo a tres dimensiones: el tipo de instrumento; la amplitud/profundidad de la evaluación practicada; 
y el énfasis ocupacional de su contenido. Cada una de las tres dimensiones se define por los indicadores que la componen. Cada indicador recibe una codificación numérica.

Procedimientos de Evaluación según Amplitud/Profundidad

001 Tamizaje/barrido

002 Interconsultas relacionadas con el usuario

003 Regreso a fuente de remisión

004 Evaluación áreas del desempeño ocupacional

005 Evaluación componentes del desempeño ocupacional

006 Retamizaje

007 Reevaluación

008 Evaluación final

009 Seguimiento

Procedimientos de Evaluación según Instrumento

010 Entrevista estructurada

011 Entrevista semiestructurada

012 Entrevista libre

013 Observación directa: individual/en grupo

014 Observación indirecta: individual/en grupo

015 Test estandarizado

016 Test formal

017 Prueba clínica

Procedimientos de Evaluación según Enfasis Ocupacional

020 Desempeño en autocuidado

021 Desempeño en actividades de trabajo/estudio/hogar 
022 Desempeño durante juego/tiempo libre

023 Componentes sensoriomotores, cognoscitivos, socioafectivos y espirituatés del desempeño ocupacional

024 Actividades funcionales: tareas sensoriales, motoras, cognoscitivas, sociales.

025 Ortesis/Prótesis/artefactos/ayudas funcionales

026 Manejo del contexto físico: casa, trabajo, estudio

027 Manejo del contexto social: familiar/educativo/laboral

\section{Procedimientos. de Tratamiento/Intervención}

Procedimientos de tratamiento/intervención son aquellas acciones propias de la terapia ocupacional, dirigidas a ejecutar y dar cumplimiento a las metas establecidas a partir de los resultados de la evaluación. Estas acciones se clasificación con base en tres dimensiones: tipo de atención; medios empleados; y áreas y componentes ocupacionales que se enfatizan. Las áreas y componentes ocupacionales guardan relación con el dominio de estudio de la terapia ocupacional (American Occupational Therapy Association, 1994; Canadian Occupational Therapists Association, 1991; Trujillo, 1987). Sin embargo,, se incorpora un nuevo concepto: el componente espiritual del desempeño. Este término apenas empieza a aparecer en la literatura profesional (Canadian Occupational Therapists Association, 1991; Hettinger, 1996; Urbanowski, 1997), no obstante estar intrínsecamente ligado al desempeño de ocupaciones. Por ejemplo, la espiritualidad subyace a la realización personal que postula el modelo «Desempeño Ocupacional Realizante» (Trujillo, 1995). 
Las tres dimensiones de procedimientos de tratamiento (tipo de atención; medios y modalidades; $y$ áreas y componentes ocupacionales) son definidas por los indicadores que las componen, los cuales reciben una codificación numérica.

Procedimientos de Tratamiento/Intervención según Tipo de Atención

031 Atención directa en institución

032 Atención directa en ambiente natural

033 Atención indirecta: a familia/cuidadores/maestros

034 Atención indirecta: plan terapéutico domiciliario/escolar/laboral

035 Educación/promoción

036 Asesorías

Procedimientos de Tratamiento/Intervención según Medios/Modalidades Empleados

040 Actividades terapéuticas individuales

041 Actividades terapéuticas de grupo

042 Relación terapéutica

043 Modalidades físicas (como técnicas de facilitación-inhibición)

044 Modalidades socioemocionales (como técnicas de remotivación y manejo del estrés)

045 Habilidades funcionales (como tareas de coordinación y destreza)

046 Adaptaciones/cambios para accesibilidad de contexto físico/social 047 Tecnología de rehabilitación

Procedimientos de Tratamiento/Intervención según Areas y Componentes Ocupacionales

Entrenar/orientar área de autocuidado:

051 comida/alimentación 
052 higiene/arreglarse

053 vestirse

054 cuidado de la salud

055 socialización

056 desempeño en casa

057 desempeño en comunidad: transporte, compras

058 respuesta a emergencias

Entrenar/orientar área de trabajo/estudio/actividades productivas:

061 mantenimiento del hogar

062 cuidado de otros

063 tareas productivas: arreglos, reparaciones, diligencias.

064 desempeño escolar

065 orientación ocupacional

066 capacitación ocupacional

067 reubicación ocupacional

Entrenar/orientar área de juego/actividades tiempo libre:

071 exploración de intereses

072 desempeño en juego/actividades tiempo libre

073 desarrollo de intereses

074 planeación y distribución del tiempo

075 entretenimiento social

Entrenar/orientar componentes sensorio motores del desempeño:

081 reconocimiento/procesamiento sensorial

082 procesamiento perceptual

083 actividad refleja

084 fuerza muscular

085 movilidad articular 
086 patrones de movimiento

087 coordinación y destreza

088 control oral/motor

089 lateralidad

090 praxis

091. tolerancia física

092 mecánica corporal

093 simplificación del trabajo/conservación de energía

Entrenar/orientar componentes cognoscitivos del desempeño:

101 estado de alerta

102 orientación temporal/espacial

102 atención/concentración

103 memoria

104 secuenciación

105 formación de conceptos

106 comprensión/conceptualización

107 resolución de problemas

108 aprendizaje

109 generalización

110 iniciación/terminación actividad

Entrenar/orientar utilización de componentes socioafectivos del desempeño:

121 motivación intrínseca

122 autoconcepto

123 intereses

124 valores

12.5 autoexpresión 


\section{6 conducta social \\ 127 capacidad adaptativa \\ 128 manejo del tiempo \\ 129 relaciones interindividuales \\ 130 integración a grupos \\ 131 autocontrol \\ 132 remotivación \\ 133 manejo del estrés}

\section{Resaltar componentes espirituales del desempeño:}

141 sentido de propósito en la vida

142 sistema de valores y creencias

143 fuentes de motivación interna

144 motivos de realización personal

Diseñar/asesorar/entrenar uso de ortesis/prótesis/tecnología de rehabilitación:

151 diseñar/asesorar/entrenar uso de ortesis

152 asesorar/entrenar uso de prótesis

153 tecnología aumentativa para el desempeño independiente (ortesis, mecanismos y aditamentos asistivos)

154 tecnología alternativa para el desempeño independiente (artefactos para movilidad, patrones de movimiento y alimentación, sillas y aparatos electrónicos/computarizados, dispositivos robóticos, computadores).

Orientar manejo/adaptación de contexto accesibles: casa, trabajo, estudio, tiempo libre: 
161 desempeño/adaptaciones/cambios en contexto familiar

162 desempeño/adaptaciones/cambios en puesto de trabajo

163 desempeño/adaptaciones/cambios en contexto escolar

164 experiencia/ajustes/reformas en contexto social

\section{Lista de Referencias}

American Occupational Therapy Association (1994), Uniform terminology for occupational therapy - Third Edition. American Journal of Occupational Therapy, 48(11), 1047-1054.

Canadian Association of Occupational Therapists (1 99 1), Occupational therapy guidelines for client-centred practice, pp. 157-159. Toronto: Autor.

Hettinger, J,(1996). Bringing spirituality into ractice. OT Week, June 13, 16-19.

Ministerio de Salud (1994). Resolución \#5261 por la cual se establece el Manual de Actividades, Intervenciones y Procedimientos del Plan Obligatorio de Salud en el Sistema General de Seguridad Social. Santa Fe de Bogotá.

Superintendencia de Salud (1997). Instrumento para la verificación de los requisitos esenciales en los prestadores de servicios de salud. Santa Fe de Bogotá: Ministerio de Salud.

Trujillo R., A. (1987). Trujillo R., A. (1987). Reflexiones sobre la Identidad del terapeuta ocupacional. Revista Acción,, 2(1),33-47.

Trujillo R., A. (1995). Desempeño ocupacional realizante: un modelo sobre los propósitos de la terapia ocupacional, Revista Ocupación Humana, 6(2), 11-22.

Urbanowski, R. (1997), Spirituality in everyday practice. OT Practice, 2(12), 18-23. 